\section{A reliable prognosis for congenital central hypoventilation syndrome should reflect a patient's genetic profile and management history}

doi:10.1038/pr.2012.99

To the Editor: The March 2012 paper titled "Progressive gray matter changes in patients with congenital central hypoventilation syndrome" by Kumar et al. commendably attempts to address long-term neurological manifestations of congenital central hypoventilation syndrome (CCHS) (1). However, as a CCHS patient (N.R.L.) and as undergraduate biomedical researchers, we believe the authors' neglect of critical aspects of the diagnosis and management of CCHS raises questions about the validity and value of their study.

CCHS is a rare autosomal dominant autonomic nervous system disorder characterized by hypoventilation, resulting from a mutation in the paired-like homeobox gene $P H O X 2 B$ $(2-5)$. A close correlation exists between genotype and phenotype, with structure-disrupting nonpolyalanine repeat expansion mutations (NPARMs) and longer polyalanine repeat expansion mutations (PARMs) giving rise to more severe and multisystemic phenotypes than shorter PARMs or structure-preserving NPARMs $(2,4)$. Proper clinical management is essential from infancy through adulthood; in particular, insufficient ventilatory support associated with hypoxia and hypercapnia inevitably translates to poor patient outcomes (6).

Kumar et al. used magnetic resonance imaging to quantify changes in gray matter in adolescent CCHS patients relative to controls after $4 \mathrm{y}$. They report that several brain regions decrease in gray matter volume in their CCHS patients whereas a limited number of other brain regions increase in gray matter volume as compared with controls. They conclude, "Patients with CCHS show progressive gray matter volume loss, after partitioning for normal age-related tissue changes in several brain regions that control autonomic, mood, motor, and cognitive functions" and infer that such tissue injury likely results from extended exposure to intermittent hypoxia (1).

The most problematic aspect of this work lies in the failure to demonstrate a direct correlation between clinical symptoms of CCHS and the causative PHOX2B mutation. Table 1 of their paper indicates that only three of seven "CCHS" patients were tested for PHOX2B mutations. Furthermore, one of those
"CCHS" patients tested negative for a $\mathrm{PHOX} 2 \mathrm{~B}$ mutation; this explicitly fails to satisfy the requirements for a definitive diagnosis of CCHS laid out in the official 2010 American Thoracic Society (ATS) Clinical Statement on CCHS (6). Although the study of Kumar et al. was initiated prior to publication of this ATS statement and adheres to the guidelines of the 1999 ATS statement, $P H O X 2 B$ had been identified as the disease-defining gene in 2003 (before initiation of this study) by several independent research groups $(2-4,7)$.

In addition, the authors do not report the specific PHOX2B mutation (NPARM or number of polyalanine repeats) for the two positively identified CCHS cases. In particular, it is instructive to recognize the disparate clinical presentations of PARMs, which range from 24 polyalanine repeats (PARs) to 33 PARs in CCHS patients and account for $90 \%$ of CCHS cases: genotypes 20/24 and occasionally 20/25 (number of alanines for each allele) frequently result in late-onset CCHS; 20/25 and some 20/26 patients require ventilation while asleep but not during waking hours (N.R.L. has the 20/25 genotype and the subsequently described phenotype); many 20/26 and nearly all 20/27 patients experience sinus pauses and require 24 -h ventilation; PARs beyond 28 alanines increase the risk of tumors of neural crest origin (2-8). Thus, given that genotype and phenotype so strongly correlate in CCHS patients, the omission of this information detracts greatly from the interpretability of the results.

The study acknowledges some of its limitations, including use of different magnetic resonance imaging scanning parameters, and geographic relocation and consequent dropout of case and control subjects. The authors also recognize that the use of only male controls is problematic in comparison with a "CCHS" group of four males and three females. However, they then conjecture that "The relative neuroprotection offered by the female sex may significantly reduce hypoxic or other injury in female adolescents." It is impossible to evaluate the potential relevance of this because the paper aggregates male and female data in CCHS patients, preempting the possibility of comparing male patients to male controls or male to female CCHS patients.

The age of the "CCHS" patients examined (mean age at first study, 16.1 y) constitutes another salient limitation left unaddressed in the study. Patients, including the cohort examined here, diagnosed early in the history of CCHS before the development and standardization of proper management techniques were more likely to have been mismanaged both clinically and by long-term caregivers $(6,8)$. This creates difficulty in distinguishing between gray matter loss due to mismanagement-induced hypoxia, including refusal of ventilatory support, and gray matter loss due to intrinsic manifestations of autonomic dysregulation.

Disregard for this hypoxia issue stems from a hidden assumption that CCHS patients inevitably face hypoxic insults throughout life. In fact, the risk for hypoxia-induced tissue 
damage has diminished significantly in recent years due to improving standards of long-term care for CCHS patients. Poignantly, although consistently managed patients thrive and mature normally, "children receiving suboptimal and inconsistent management were left with significant disabilities, and achievement of lesser accomplishments..." (8). Thus, genotype-phenotype, age, gender, and management status of subjects must be carefully considered when interpreting data from CCHS patients. Singular approaches to research on diseases of autonomic dysregulation risk obscuring their complexity and variability while precluding personalized treatment.

\section{Naomi R. Latorraca ${ }^{1}$ and Rohith Palli ${ }^{2}$}

${ }^{1}$ Undergraduate major in molecular biology and history, University of Pittsburgh, Pittsburgh, Pennsylvania; ${ }^{2}$ Undergraduate major in mathematics, University of Pittsburgh, Pittsburgh, Pennsylvania. Correspondence: E-mail: Naomi R. Latorraca (nrl23@pitt.edu)

\section{REFERENCES}

1. Kumar R, Woo MS, Macey PM, Woo MA, Harper RM. Progressive gray matter changes in patients with congenital central hypoventilation syndrome. Pediatr Res 2012;71:701-6.
2. Matera I, Bachetti T, Puppo F, et al. PHOX2B mutations and polyalanine expansions correlate with the severity of the respiratory phenotype and associated symptoms in both congenital and late onset central hypoventilation syndrome. J Med Genet 2004;41:373-80.

3. Sasaki A, Kanai M, Kijima K, et al. Molecular analysis of congenital central hypoventilation syndrome. Hum Genet 2003;114:22-6.

4. Weese-Mayer DE, Berry-Kravis EM, Zhou L, et al. Idiopathic congenital central hypoventilation syndrome: analysis of genes pertinent to early autonomic nervous system embryologic development and identification of mutations in PHOX2b. Am J Med Genet A 2003;123A:267-78.

5. Trochet D, O’Brien LM, Gozal D, et al. PHOX2B genotype allows for prediction of tumor risk in congenitalcentral hypoventilation syndrome. Am J Hum Genet 2005;76:421-6.

6. Weese-Mayer DE, Berry-Kravis EM, Ceccherini I, Keens TG, Loghmanee DA, Trang H; ATS Congenital Central Hypoventilation Syndrome Subcommittee. An official ATS clinical policy statement: Congenital central hypoventilation syndrome: genetic basis, diagnosis, and management. Am J Respir Crit Care Med 2010;181:626-44.

7. Amiel J, Laudier B, Attié-Bitach T, et al. Polyalanine expansion and frameshift mutations of the paired-like homeobox gene PHOX2B in congenital central hypoventilation syndrome. Nat Genet 2003;33:459-61.

8. Weese-Mayer DE, Rand CM, Berry-Kravis EM, et al. Congenital central hypoventilation syndrome from past to future: model for translational and transitional autonomic medicine. Pediatr Pulmonol 2009; 44:521-35.

medical benefits. The reasons for declining a request varied, but included statements often found in response to testing for other life-threatening diseases, and included parental refusal. We had genetic information from a limited number of CCHS subjects (two positive (20/27 and 20/25 polyalanine expansions), one negative, and four untested); based on other published studies, from 62 to $97 \%$ of CCHS subjects show PHOX2B mutations $(5,6)$, and we believe that the majority of our CCHS subjects also have such mutations, along with the primary CCHS characteristics described above. However, a small proportion of CCHS subjects in the literature shows an absence of $P H O X 2 B$ mutation, despite presence of characteristics positive for the syndrome (5). Although handicapped by largely absent specific genetic information, we used the obvious clinical presentation here.

We did raise several study limitations in the article, including limited numbers, the proportion of male subjects, and slightly variable MRI procedures between the 4-y intervals. However, those issues should not significantly affect the findings. Subject relocation, especially of controls, reduced numbers, but the design using CCHS and control subjects as their own controls was necessary to control for normal regional gray matter changes over time, and to substantially assist statistical assessment.

The second major concern of Latorraca and Palli is the suggestion that we fail to consider the potential for ventilatory mismanagement as contributing to the enhanced injury during the 4-y scanning interval. However, we indeed raised the issue that the progression of injury develops from hypoxic exposure rather than consequences of PHOX2B mutation (page 4, lines 8-25). The parents of our subjects were extremely motivated, as evidenced by their willingness to transport their charges for scanning across the continent, and were very much aware of the 